ARTICLE

https://doi.org/10.1038/s41467-019-12115-7

\title{
A highly conductive fibre network enables centimetre-scale electron transport in multicellular cable bacteria
}

Filip J.R. Meysman (10) 1,2, Rob Cornelissen (10) ${ }^{3}$, Stanislav Trashin (1) ${ }^{4}$, Robin Bonné (i) ${ }^{3}$, Silvia Hidalgo Martinez ${ }^{1}$, Jasper van der Veen ${ }^{5}$, Carsten J. Blom ${ }^{6}$, Cheryl Karman (1) 1,4, Ji-Ling Hou ${ }^{3}$, Raghavendran Thiruvallur Eachambadi (10 ${ }^{3}$, Jeanine S. Geelhoed (10 ${ }^{1}$, Karolien De Wael ${ }^{4}$, Hubertus J.E. Beaumont ${ }^{6}$, Bart Cleuren (10 ${ }^{7}$, Roland Valcke ${ }^{8}$, Herre S.J. van der Zant (i) ${ }^{5}$, Henricus T.S. Boschker (1) ${ }^{1,2} \&$ Jean V. Manca ${ }^{3}$

Biological electron transport is classically thought to occur over nanometre distances, yet recent studies suggest that electrical currents can run along centimetre-long cable bacteria. The phenomenon remains elusive, however, as currents have not been directly measured, nor have the conductive structures been identified. Here we demonstrate that cable bacteria conduct electrons over centimetre distances via highly conductive fibres embedded in the cell envelope. Direct electrode measurements reveal nanoampere currents in intact filaments up to $10.1 \mathrm{~mm}$ long (>2000 adjacent cells). A network of parallel periplasmic fibres displays a high conductivity (up to $79 \mathrm{~S} \mathrm{~cm}^{-1}$ ), explaining currents measured through intact filaments. Conductance rapidly declines upon exposure to air, but remains stable under vacuum, demonstrating that charge transfer is electronic rather than ionic. Our finding of a biological structure that efficiently guides electrical currents over long distances greatly expands the paradigm of biological charge transport and could enable new bio-electronic applications.

\footnotetext{
${ }^{1}$ Department of Biology, University of Antwerp, Universiteitsplein 1, B-2610 Wilrijk, Belgium. ${ }^{2}$ Department of Biotechnology, Delft University of Technology, Van der Maasweg 9, $2629 \mathrm{HZ}$ Delft, The Netherlands. ${ }^{3}$ X-LAB, Hasselt University, Agoralaan D, B-3590 Diepenbeek, Belgium. ${ }^{4}$ AXES Research group, Department of Chemistry, University of Antwerp, Groenenborgerlaan 171, B-2020 Antwerpen, Belgium. ${ }^{5}$ Department of Quantum Nanoscience, Kavli Institute of Nanoscience, Technical University Delft, Lorentzweg 1, 2628 CJ Delft, The Netherlands. ${ }^{6}$ Department of Bionanoscience, Kavli Institute of Nanoscience, Delft University of Technology, van der Maasweg 9, 2629 HZ Delft, The Netherlands. ${ }^{7}$ Theoretical Physics, Hasselt University, Agoralaan D, B-3590 Diepenbeek, Belgium. ${ }^{8}$ Molecular and Physical Plant Physiology, Hasselt University, Agoralaan D, B-3590 Diepenbeek, Belgium. Correspondence and requests for materials should be addressed to F.J.R.M. (email: filip.meysman@uantwerpen.be)
} 
C harge transfer is fundamental to life, and organisms have evolved various conductive structures to support vital processes, such as enzymatic catalysis, photosynthesis and cellular respiration ${ }^{1-5}$. The distance of biological charge transport has long been thought to be limited to the micrometre scale ${ }^{6-8}$. However, this idea has been recently been challenged by observations on cable bacteria, which hint at electrical currents running through centimetre-long multicellular filaments ${ }^{9-13}$. Cable bacteria grow in marine $\mathrm{e}^{14,15}$ and freshwater sediments ${ }^{16,17}$ by coupling the oxidation of an electron donor (sulphide) on one end of the filament to the reduction of an electron acceptor (oxygen, nitrate) on the opposite end ${ }^{10}$. This necessitates that these multicellular bacteria channel an electrical current along their centimetre-long filaments. Multiple lines of indirect evidence $^{10,11,18-20}$ suggest that this current is guided internally from cell to cell along the longitudinal axis of the filaments, but attempts to directly quantify this current have been unsuccess$\mathrm{ful}^{10}$, and the nature of the charge carriers (electronic or ionic) remains unknown ${ }^{13}$. Here, we present electrical and electrochemical measurements that uncover the pathway and magnitude of the electrical currents inside cable bacteria.

\section{Results}

Electrical measurements on cable bacterium filaments. We speculated that previous attempts to measure currents might have been unsuccessful due to degradation or instability of filaments after isolation from anaerobic sediments. To avoid this, we measured conductivity on filaments that were freshly retrieved from sediment enrichment cultures, immediately deposited onto electrodes having two metal contacts separated by a nonconductive interspacing $\Delta x$, and quickly air-dried without any further chemical fixation (Fig. 1). Current measurements were initiated within 5-15 min after filament harvesting and performed in ambient air at a constant bias $\Delta V=100 \mathrm{mV}$. This voltage bias was selected to avoid Faradaic processes at the electrodes (e.g. the electrolysis of water), as well as to be representative for the in vivo situation. Raman spectroscopy on living cable bacteria reveals a voltage drop of $\sim 12-15 \mathrm{mV} \mathrm{mm}^{-1}$ of filament ${ }^{10}$, thus corresponding to a total voltage drop $\Delta V=1.2-150 \mathrm{mV}$ for the filament lengths investigated here $(\Delta x=0.1-10 \mathrm{~mm})$. The selected $\Delta V=100 \mathrm{mV}$ lies at the upper end of this range and was consistently used in all measurements. Observed currents rapidly decreased with time, and depended on the non-conductive interspacing $\Delta x$. Therefore, initial currents were normalized as $I_{\text {norm }}=I_{\max }{ }^{\star}\left(\Delta x / \Delta x_{\text {ref }}\right)$ with $\Delta x_{\text {ref }}=300 \mu \mathrm{m}$ to allow comparison between different measurements.

Individual cable bacterium filaments were found to be intrinsically conductive and showed normalized initial currents up to $2.7 \mathrm{nA}(N=47$; Supplementary Data 1$)$. To verify that currents were flowing through the cable bacteria, filaments were
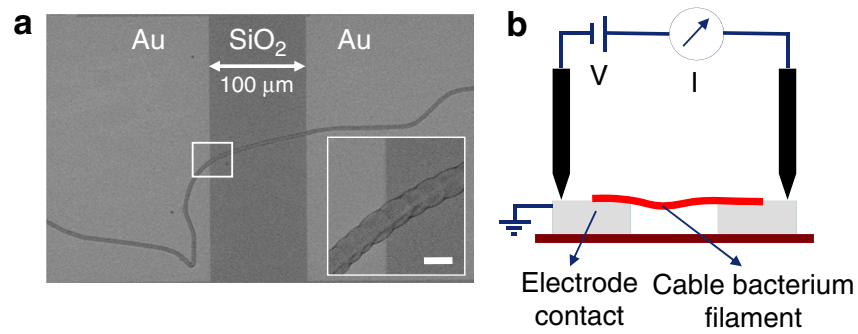

Fig. 1 Current measurements on individual cable bacterium filaments. a SEM image $(\times 470)$ of an individual air-dried cable bacterium filament deposited on a non-conductive $\mathrm{SiO}_{2}$ substrate with $\mathrm{Au}$ contact pads. Inset: zoom of intact cable bacterium filament $(\times 11,500)$. Scale bar: $5 \mu \mathrm{m}$. b Schematic of conductance measurement suspended in air between two elevated contact pads. When filaments were cut with a scalpel, the current ceased immediately (Fig. 2a; Supplementary Movie 1). For reference, filaments of Thiofilum flexile, another aquatic multicellular bacterium, were subjected to the same electrical characterization. Currents mediated by $T$. flexile were 5 orders of magnitude lower (Supplementary Fig. 1), showing that the conductivity of cable bacteria is exceptional and not a general property of filamentous bacteria. Voltage contrast imaging on grounded interdigitated electrodes confirmed that cable bacteria efficiently dissipate charges, while this was not the case for T. flexile (Fig. 3).

When filaments were examined under ambient air, the current decreased by an order of magnitude within the first $10 \mathrm{~min}$, and only $\sim 2 \%$ of the initial current remained after $30 \mathrm{~min}$ (Fig. 4a). This precluded the collection of stable current $(I) /$ voltage $(V)$ curves. Similar results were obtained when filaments were only intermittently exposed to the same voltage bias, indicating that the current decay is not linked to cumulative electron transport. However, when current measurements were executed under an $\mathrm{N}_{2}$ atmosphere, the decrease in conductance was substantially slower, and upon sudden exposure to air, the conductance rapidly decreased (Fig. 2b). This suggests a progressive $\mathrm{O}_{2}$-induced degeneration of crucial components in the conductive pathway of the filaments. Occasionally, we encountered filaments that showed no conduction, and upon visual inspection, physically damaged segments were sometimes noticeable along these filaments, likely caused by filament manipulation. When these "bad sections" were bridged using water-based carbon paste, the conductance of the filament could be reconstituted (Fig. 5). Consequently, damage during filament retrieval, combined with improper electrode connections and fast oxidative ageing under ambient air conditions, could explain why previous attempts have failed to directly measure electrical currents in cable bacterium filaments ${ }^{10}$.

In a subsequent set of experiments, currents were measured on single cable bacterium filaments under an $\mathrm{N}_{2}$ atmosphere using electrode contacts enhanced with carbon paste (Supplementary Data 2). This way, stable $I / V$ curves could be collected, which were highly linear and symmetric over $-0.1 \mathrm{~V}$ to $0.1 \mathrm{~V}$ range (Fig. 6b), and the resistance $R$ was calculated from the slope of the $I / V$ curve at the origin. Normalized currents $\left(I_{\text {norm }}=(\Delta V / R)\right.$ ${ }^{*}\left(\Delta x / \Delta x_{\text {ref }}\right)$ with $\Delta V=0.1 \mathrm{~V}$ and $\left.\Delta x_{\text {ref }}=300 \mu \mathrm{m}\right)$ now ranged up to $300 \mathrm{nA}$ (Supplementary Data $2 ; N=32$ ), corresponding to a resistance of as low as $5 \mathrm{k} \Omega$ over a single cell (Fig. 6c). In consecutive experiments, the non-conductive spacing $\Delta x$ was systematically increased, and the longest conductive filament length attained was $\Delta x=10.1 \mathrm{~mm}$ (Fig. 7; longer filament lengths were not tested). Electrical currents were hence guided across a sequence of $>2000$ cells (mean cell length $4.95 \mu \mathrm{m}$; ref. ${ }^{21}$ ). This largely exceeds the maximal distance over which biological electron transport has been previously observed, which is the micrometre scale as documented for the "nanowire" appendages in metal-reducing bacteria such as Geobacter ${ }^{6}$ and Shewanella ${ }^{7,8}$.

The conductive structures of cable bacteria. In order to facilitate the observed currents, cable bacteria must contain suitably conductive structures. Previous work has revealed that the periplasm of cable bacteria contains a network of parallel fibres ${ }^{10,21,22}$, which are continuous across cell-cell junctions. Because this periplasmic fibre network runs across cells along the whole filament, it has been proposed to form the conductive conduit enabling long-distance electron transport ${ }^{10}$. To test this hypothesis, we developed an SDS/EDTA extraction procedure that removes the cytoplasm and membranes, leaving behind a $60 \mathrm{~nm}$ thick periplasmic sheath that is made up of the fibre network ${ }^{21}$ 

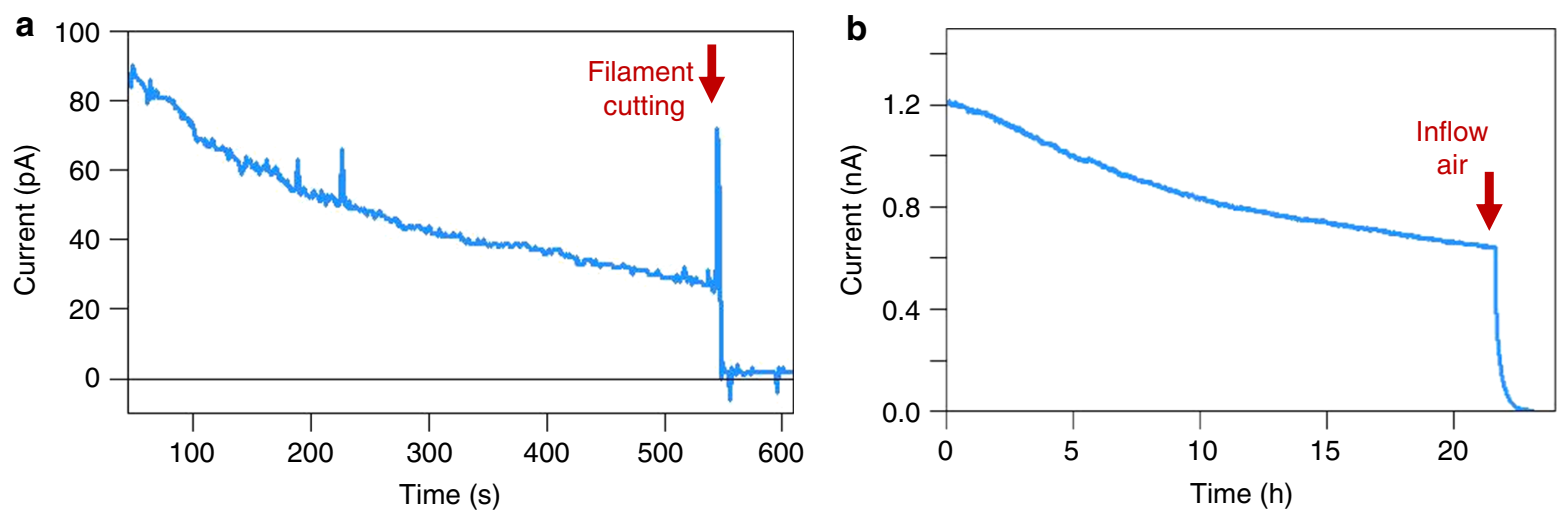

Fig. 2 Current response of intact cable bacterium filaments. a Time evolution of the current response of an intact cable bacterium filament under ambient air. Current recording at a constant bias of $100 \mathrm{mV}$. Physical disruption immediately halts the current at the time point indicated by the red arrow. $\mathbf{b}$ Current recording of an intact filament under $\mathrm{N}_{2}$ atmosphere in a glove box (constant bias of $100 \mathrm{mV}$ ). At the time point of the red arrow, the glove box is opened and air flows in
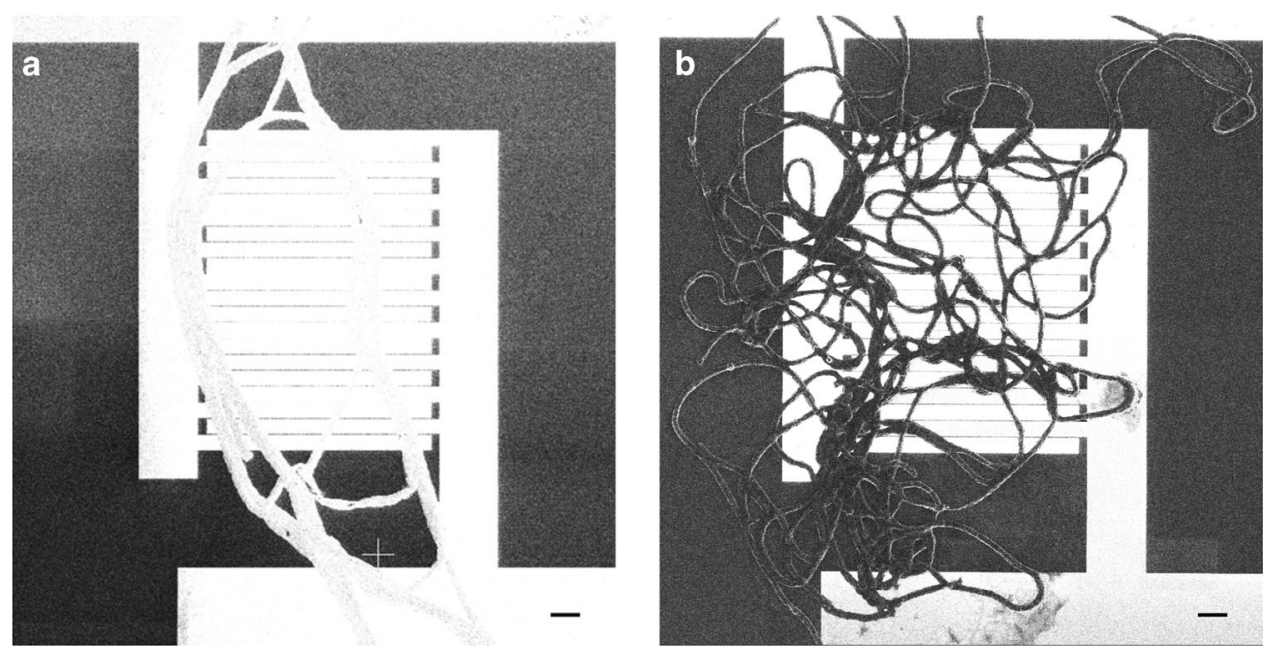

Fig. 3 Passive voltage contrast (PVC) imaging of filamentous bacteria on interdigitated electrodes. a PVC image of a bundle of cable bacterium filaments. b PVC image of a bundle of filaments of the filamentous bacterium Thiofilum flexile. Surfaces that are capable of discharging charges faster appear brighter during PVC. The electrodes, as well as the cable bacteria, are brighter, but not T. flexile. Scale bars are $10 \mu \mathrm{m}$
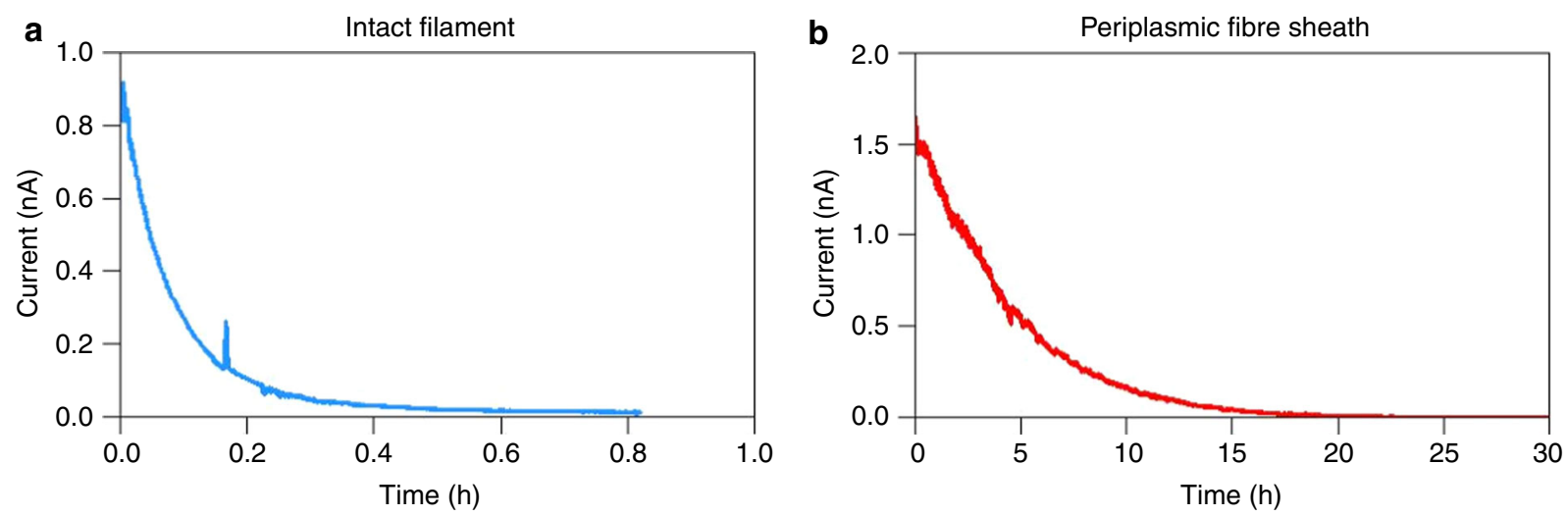

Fig. 4 Temporal decrease of currents in intact filaments and fibre sheaths. a Representative current response of an intact cable bacterium filament recorded under ambient air at room temperature at a fixed bias of $0.1 \mathrm{~V}$. b Representative current response of a periplasmic fibre sheath recorded under ambient air at room temperature at a fixed bias of $0.1 \mathrm{~V}$. Note the difference in time scale between both panels

(Fig. 6d). When subjected to the same current measurement procedure, these periplasmic fibre sheaths showed resistances and normalized currents similar to those mediated by intact filaments (Fig. 6e; Supplementary Data 3, N=28). This indicates that the electrical current most likely runs through the periplasmic fibres, which make up two-thirds of the volume of the periplasmic fibre sheath $^{21}$. Strikingly, the current decay in ambient air was much slower (reduction of $50 \%$ in $5 \mathrm{~h}$; Fig. $4 \mathrm{~b}$ ) than for intact cable 
a

Before

Two terminal carbon paste contacts
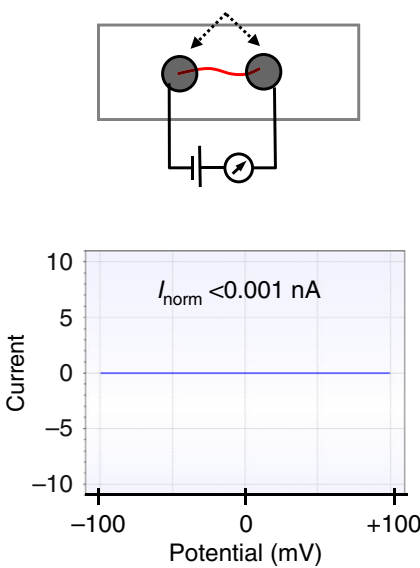

b

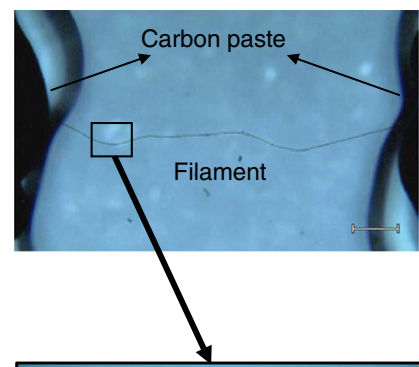

C

After

Add carbon paste across bad section
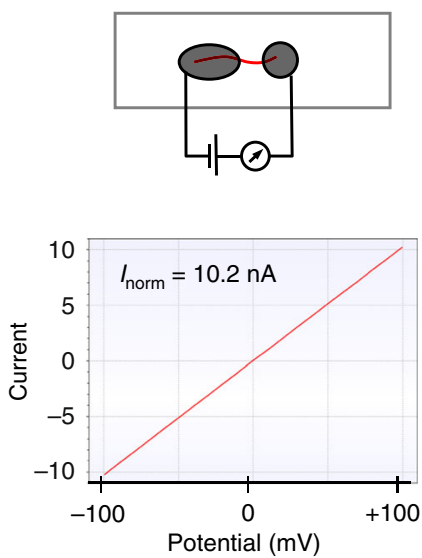

Fig. 5 Damaged filaments that are non-conductive can become conductive upon repair. a Initial situation: a current measurement is conducted on a filament interfaced at the terminal ends via two dots of carbon paste. The $I / V$ curve shows no measurable current. $\mathbf{b}$ Visual inspection of the filament reveals a physically damaged segment. Scale bar upper panel $=200 \mu \mathrm{m}$. Scale bar lower panel $=20 \mu \mathrm{m}$. c Situation after filament repair: the physically damaged segment is covered by carbon paste. The $I / V$ curve now reveals a high conductance. $I_{\text {norm }}=$ normalised current (see definition in the main text)
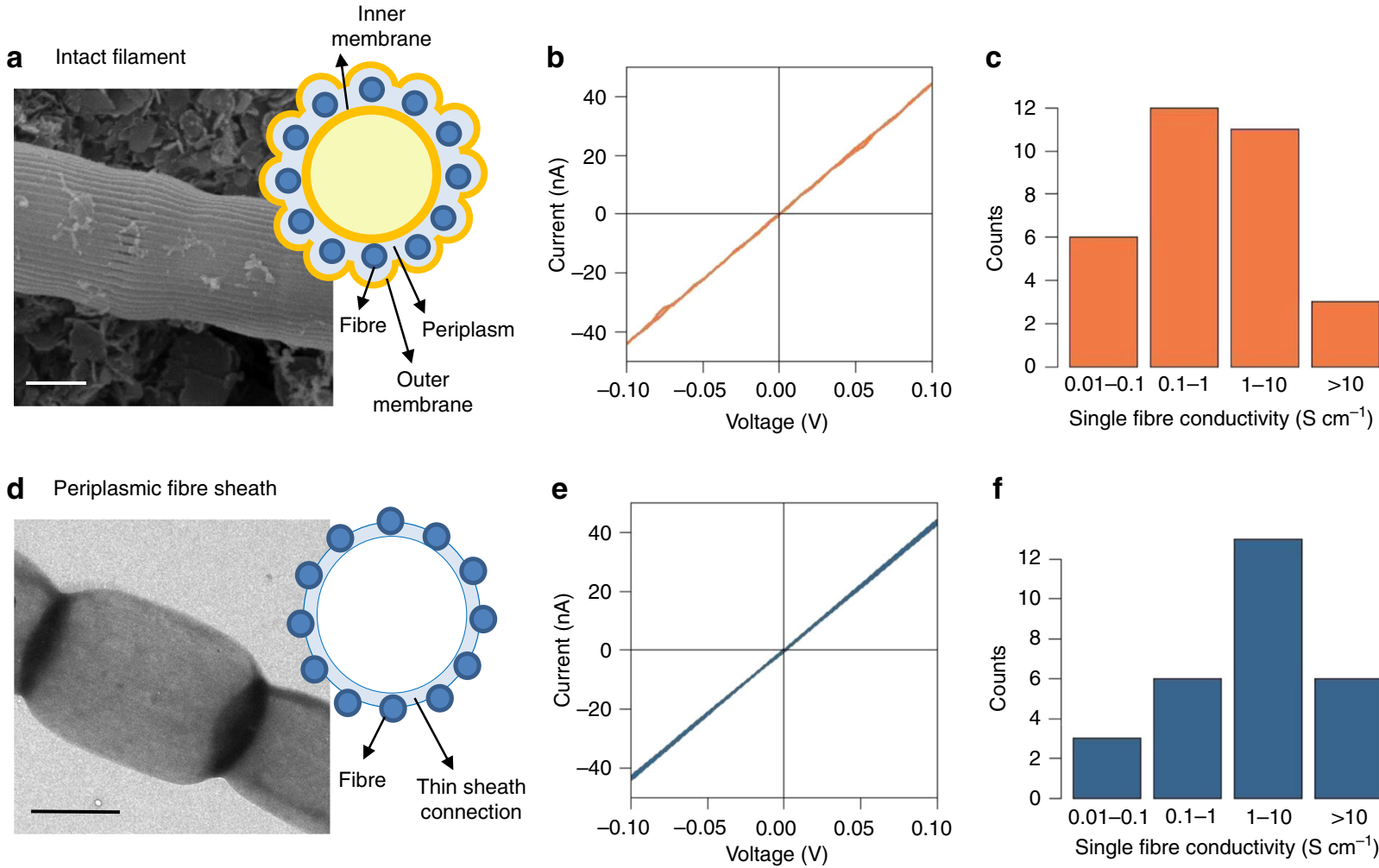

$\mathbf{f}$

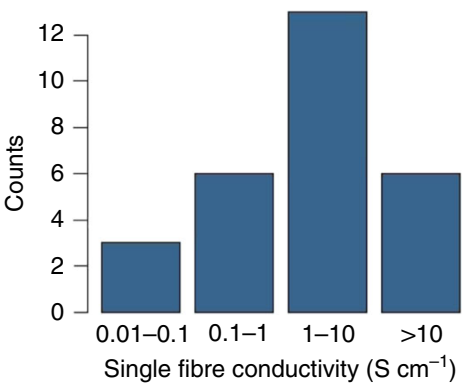

Fig. 6 Conductance measurements on individual cable bacterium filaments. a SEM image $(\times 7500)$ of an intact cable bacterium filament with schematic of the structure in cross-section revealing the periplasmic embedding of the fibres (blue circles). Scale bar $=1 \mu \mathrm{m}$. $\mathbf{b}$ Representative $I / \mathrm{V}$ curve of an intact filament recorded under $\mathrm{N}_{2}$ atmosphere $\left(\Delta x=250 \mu \mathrm{m}\right.$; scan rate $\left.10 \mathrm{mV} \mathrm{s}^{-1}\right)$. Forward and reverse scans are plotted, the current is normalized as $I^{\star} \Delta x / \Delta x_{\text {ref }}$ with $\Delta x_{\text {ref }}=300$. c Histogram of whole filament conductivities recorded ( $N=32$ specimens). d TEM image of an extracted cable bacterium filament retaining the fibre sheath with schematic of cross-section. Scale bar $=2 \mu \mathrm{m}$. e Representative I/V curve of a periplasmic fibre sheath recorded under $\mathrm{N}_{2}$ atmosphere $\left(\Delta x=450 \mu \mathrm{m}\right.$; scan rate $\left.10 \mathrm{mV} \mathrm{s}^{-1}\right)$. Forward and reverse scans are plotted, the current is normalized as $I^{\star} \Delta x / \Delta x_{\text {ref }}$ with $\Delta x_{\text {ref }}=300$. f Histogram of single fibre conductivities recorded ( $N=28$ specimens) 
bacteria, thus indicating that the conductance of the periplasmic fibre sheath is less susceptible to oxidative ageing. Furthermore, when two-probe current measurements were repeated in a high vacuum $\left(<10^{-7}\right.$ bar $)$ pressure chamber, the conductance of the fibre sheath remained stable over a period of weeks (Fig. 8b). Moreover, four-probe measurements on a single fibre sheath stretched over a sequence of 10 consecutive gold contacts with $\Delta x=100 \mu \mathrm{m}$ interspacing, revealed that the contact resistance accounts for $5-60 \%$ of the total resistance (Fig. 8c). This indicates that the conductivity values derived from two-probe measurements (as presented in Fig. 6) are conservative estimates.

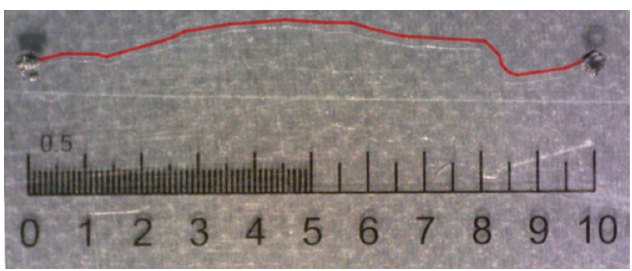

Fig. 7 Micrograph of a conductive long individual cable bacterium filament (thin white thread-the red line traces the filament) connected by carbon paste electrode connections (black dots). The scale ranges from 0 to 10 millimetres. The straight line distance between the electrodes is $9.7 \mathrm{~mm}$. The filament length is $10.1 \mathrm{~mm}$

The conductive pathway in cable bacteria. The fact that intact cable bacterium filaments can be interfaced with electrodes indicate there must be an "outward" conductive conduit that enables a current across the outer cell membrane and is internally linked to the periplasmic fibres. Examination of intact cable bacterium filaments by cyclic voltammetry on gold electrodes modified with a mercaptohexanol self-assembled layer reveals the presence of redox sites with a reduction potential $\left(E^{\mathrm{O}^{\prime}}\right)$ around $+0.155 \mathrm{~V}$ vs. SHE (Fig. 9b) that matches the value determined by differential pulse voltammetry (Fig. 9b, inset). The linear dependence of the cathodic peak current on the scan rate (Supplementary Fig. 2) indicates that these redox sites are not freely diffusible co-factors, but must be linked to the surface of the filaments, and so these redox sites could be part of outward electron conduits enabling external electron transfer (Fig. 9a). We propose that the fast decrease in conductance observed for intact filaments in air (Fig. 4a) is likely caused by oxidative decay of these outward electron conduits. The slower oxidative ageing of the periplasmic fibre sheath (Fig. 4b) suggests that it consists of a different material than the outward conduits. This hypothesis is supported by resonance Raman microscopy, which reveals the signature of cytochromes in intact cable bacteria ${ }^{11}$, allowing the possibility that cytochromes could be part of the outward electron conduits. In contrast, cyclic voltammetry of the periplasmic fibre sheath does not show any redox behaviour (Supplementary Fig. 2), while the Raman signature of cytochromes is also entirely absent (Fig. 8c). Accordingly, electron transport along heme
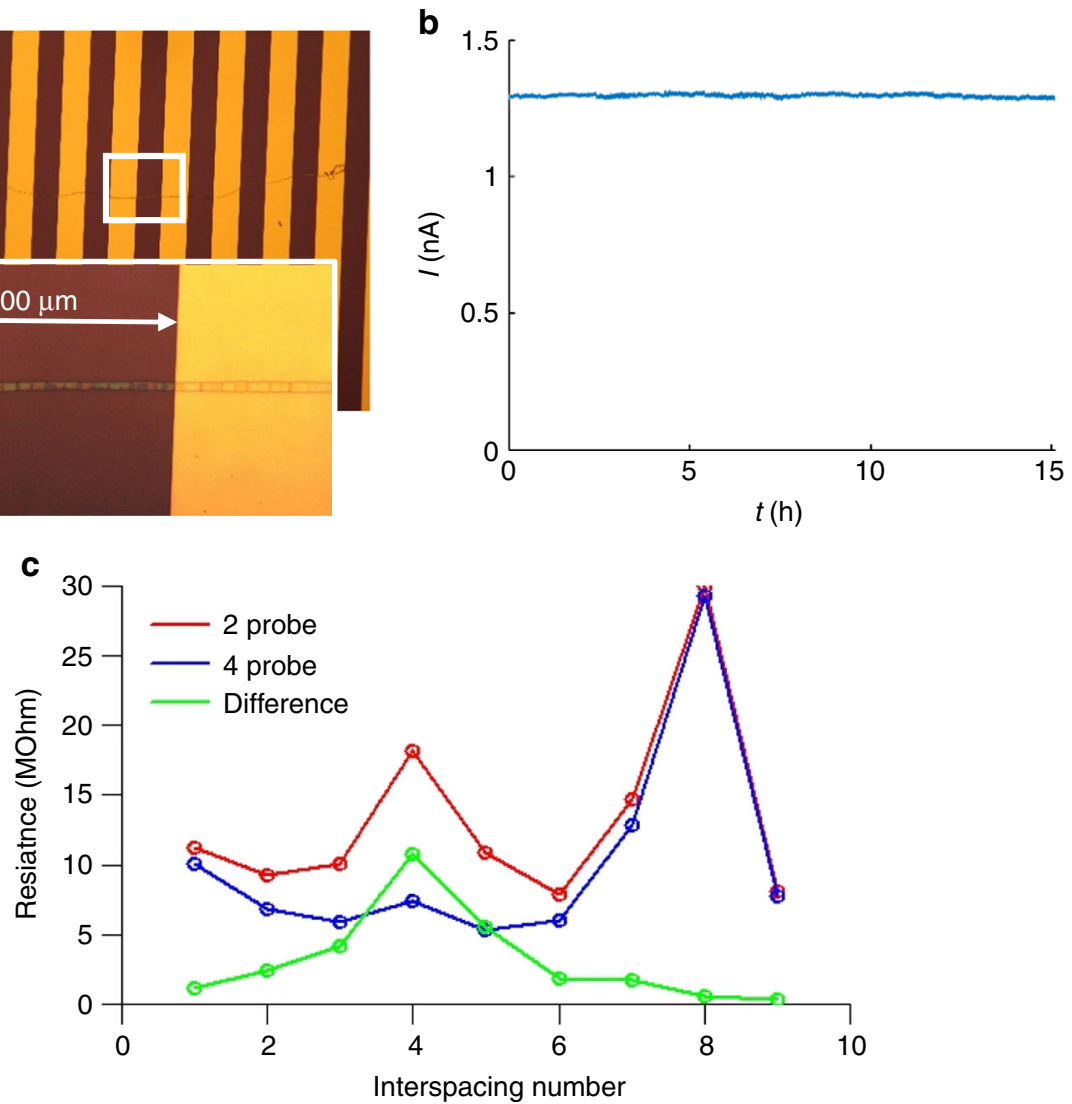

Fig. 8 Contact resistance measurements under vacuum. a Micrograph of a single periplasmic fibre sheath on a $\mathrm{SiO}_{2}$ electrode stretched across 11 consecutive Au contacts (each $100 \mu \mathrm{m}$ wide) separated by non-conductive interspacings (each $100 \mu \mathrm{m}$ wide). The inset shows a close-up of one bridged interspacing defined by the white rectangle. $\mathbf{b}$ Long-term current measurement across a single non-conductive interspacing at constant bias of $100 \mathrm{mV}$. The resistance across this non-conductive interspacing was repeatedly measured over a 4-week span and remained within $10 \%$ of the initial value. c Resistance across nine consecutive non-conductive interspacings as measured by two-probe (red line) and four-probe (blue) methods. The difference (green line) represents the contact resistance 
a
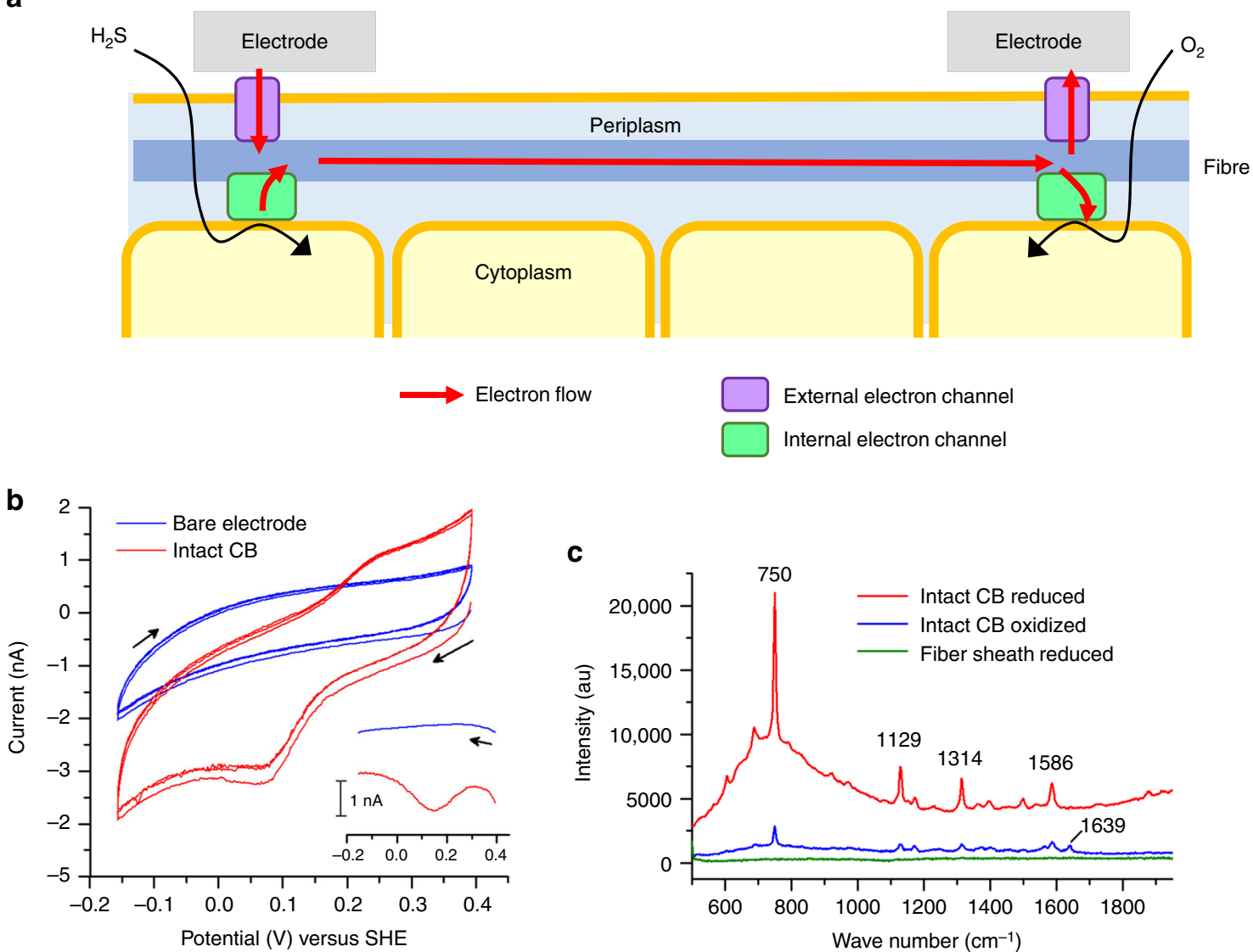

Fig. 9 Mechanism of electron transport through cable bacterium filaments. a Schematic of how external electron transport (EET) and long-distance electron transport (LDET) are combined in a cable bacterium filament. b Cyclic (main) and differential pulse (inset) voltammograms of intact cable bacteria (CB) at a gold disk electrode premodified by mercaptohexanol. Voltammograms are recorded in PBS ( $\mathrm{pH} 7.4$ ) purged with $\mathrm{N}_{2}$ with a scan rate $0.02 \mathrm{Vs}{ }^{-1}$. The bare electrode has no cable bacteria filaments, but is modified with the same mercaptohexanol self-assembled monolayer. Potential is displayed versus standard hydrogen electrode (SHE). Inset: differential pulse voltammetry shows that redox sites have a reduction potential $\left(E^{\circ}\right)$ near $+0.155 \mathrm{~V}$ vs. $\mathrm{SHE}$, which is consistent with the peak-to-peak separation in the cyclic voltammogram. c Resonance Raman spectra of intact cable bacterium filaments (reduced and oxidized) and periplasmic fibre sheath (reduced). Intact cable bacteria show typical spectra for c-type cytochromes as recorded in ref. ${ }^{11}$. The periplasmic fibre sheath spectra do not show any sign of cytochromes. All presented spectra were recorded with the same settings and are averages of $N=10$ spectra corrected for background

groups in cytochromes cannot explain long-distance conduction along the fibre structure.

\section{Discussion}

We have succeeded into depositing intact cable bacterium filaments onto micro-fabricated electrodes, in such a way that the filaments remain conductive. These results unequivocally demonstrate that electrical currents are running through cable bacteria. The observation that intact cable bacterium filaments can be interfaced with electrodes (Fig. 9a) also suggests that they are capable of performing extracellular electron transfer (EET), a capability that has so far only been reported for unicellular bacteria ${ }^{6,8}$. The physiological benefit of EET for cable bacteria is unclear, but it could explain their presence near the anode of sediment batteries ${ }^{23}$ and might allow direct interspecies electron transfer with other microbes. The latter has recently been invoked to explain anomalous isotope labelling data in sediments with cable bacteria activity 24 .

Our data additionally suggest that periplasmic fibres in the cell envelope are the conductive conduits in cable bacteria, and they provide insight into the nature of the charge transport mechanism inside these conductive structures. If electrical currents were due to ionic conduction, the current would substantially drop under vacuum conditions, as water is removed from the sample and ion mobility is greatly reduced ${ }^{25}$. Ionic conduction in a surrounding water film or charge transfer through ion-conductive polymers in the filament would also rapidly lead to charge accumulation at electrodes ${ }^{25}$, which is not observed. The fact that we observe a sustained (direct current) conductivity under vacuum (Fig. $8 \mathrm{~b}$ ) thus provides a strong indication that electrical currents through cable bacteria are electronic in nature, and so charge carriers must either be electrons or holes. Electronic charge transport is fully consistent with the metabolism of cable bacteria ${ }^{10}$, which involves the distant coupling of two redox halfreactions by a flow of electrons from the electron donor sulphide to the electron acceptors such as oxygen or nitrate ${ }^{13,20}$ (Fig. 9a).

Our data also allow a first assessment of the electrical properties of the conductive structures in cable bacteria, demonstrating that the periplasmic fibres have a high conductivity and are able to sustain a high current density. FIB-SEM imaging shows $N_{\mathrm{F}}=59-61$ fibres per filament, and a mean fibre diameter of $\delta_{\mathrm{F}}=50 \pm 7 \mathrm{~nm}$ for the filaments used in the conductivity measurements ${ }^{21}$. Assuming that the fibres are cylindrical (cross-sectional area $A_{\mathrm{F}}=\pi d_{\mathrm{F}}^{2} / 4$ ) and solely responsible for the electron transport, our periplasmic fibre sheath data reveal that individual 
fibres can conduct normalized currents $I_{\mathrm{F}}=I_{\text {norm }} / N_{\mathrm{F}}$ up to $1.3 \mathrm{nA}$ (Supplementary Data 3) at a corresponding current density $J_{\mathrm{F}}=$ $I_{\mathrm{F}} / A_{\mathrm{F}}$ of $6.5 \times 10^{5} \mathrm{~A} \mathrm{~m}^{-2}$, which is comparable to the current density in household copper wiring $\left(\sim 10^{6} \mathrm{~A} \mathrm{~m}^{-2}\right)$. This translates into a single fibre conductivity up to $\sigma_{\mathrm{F}}=20.1 \mathrm{~S} \mathrm{~cm}^{-1}$ (Fig. 6f; Supplementary Data 3), which is nearly 1000 times higher than values estimated for Shewanella nanowires $\left(0.03 \mathrm{~S} \mathrm{~cm}^{-1}\right.$; ref. $\left.{ }^{26}\right)$ and a magnitude higher than values reported for wild-type Geobacter pili $\left(0.05-1.5 \mathrm{~S} \mathrm{~cm}^{-1}\right.$; refs. 27,28$)$. Likewise, if we also estimate single fibre conductivities from the intact filament data (Supplementary Data 2), we obtain a similar frequency distribution of high fibre conductivities ranging up to $79 \mathrm{~S} \mathrm{~cm}^{-1}$ (Fig. 6c).

The conductivities for the periplasmic fibres in cable bacteria are the highest reported so far for any natural biological material, and substantially exceed the conductivity of pristine conductive polymers used in organic electronics ${ }^{29}$. This implies that biological evolution has produced a highly conductive organic structure, enabling electron transfer across centimetre-scale distances with low dissipative loss. A deeper investigation of the molecular structure and electrical properties of the periplasmic fibres in cable bacteria is needed to resolve the conduction mechanism and explore the potential of the conductive fibres as a basis for future bio-inspired electronics. Additionally, the combination of EET and long-distance electron transport (LDET) in a single organism may provide new opportunities for bio-electrical systems, as electrons can be channelled from further away, alleviating the accumulation of microbial metabolites near electrodes.

\section{Methods}

Bacterial strains. Cable bacteria were enriched from surface marine sediment collected from a creek bed within the Rattekaai salt marsh, The Netherlands $\left(51^{\circ} 26^{\prime}\right.$ $\left.21^{\prime \prime} \mathrm{N}, 04^{\circ} 10^{\prime} 11^{\prime \prime} \mathrm{E}\right)$. Sediment was sieved, homogenized, repacked into PVC core liner tubes (diameter $40 \mathrm{~mm}$, height $100 \mathrm{~mm}$ ), and incubated with overlying aerated artificial seawater (salinity 30 , temperature $20^{\circ} \mathrm{C}$ ). When the sediment showed the distinct geochemical fingerprint of electrogenic sulphur oxidation, it was used for the retrieval of cable bacterium filaments. Thiofilum flexile EJ2M-B ${ }^{\mathrm{T}}$ was obtained from the German Collection of Microorganisms and Cell cultures (DSMZ, Braunschweig, Germany) and cultured in defined liquid medium at a temperature of $27^{\circ} \mathrm{C}$.

Filament extraction. Cable bacterium filaments were gently pulled from the top layer of the sediment enrichments with custom-made glass hooks. After transfer to a drop of purified water (ISO 3696 Grade 1, MilliQ) on a glass microscope coverslip, filaments were subjected to a sequence of washes and chemical extractions. "Intact" cable bacterium filaments were washed 4-6 times in MilliQ droplets to remove any surrounding sediment and debris. To extract the fibre sheath, washed cable bacteria filaments were first incubated for $10 \mathrm{~min}$ at room temperature (RT) in a droplet of $1 \%(\mathrm{w} / \mathrm{w})$ aqueous solution of sodium dodecyl sulphate (SDS), followed by six MilliQ washes. Specimens were subsequently transferred to a droplet of $1 \mathrm{mM}$ aqueous solution of sodium ethylene diamine tetra acetic acid (EDTA, pH 8), incubated for $10 \mathrm{~min}$ at RT, and finally washed six times in MilliQ. The extraction treatment removes the cytoplasm and membranes while retaining a thin sheath that includes the periplasmic fibres, as described in detail in ref. ${ }^{21}$.

Electrical measurements. Single filaments or bundles of filaments were deposited after extraction onto custom-built electrodes consisting of a non-conductive substrate $\left(\mathrm{SiO}_{2}\right.$, mica or glass) with two pre-patterned conductive contact pads separated by a non-conductive interspacing $(\Delta x=66-9700 \mu \mathrm{m})$. Filaments were air-dried ( $\sim 5 \mathrm{~min})$ before conductivity measurements started. Contact pads were obtained by $\mathrm{Au}$ or $\mathrm{Sn}$ deposition onto the substrate or by positioning carbon paste droplets (EM-Tec C30) on the terminal ends of filaments. Conductance measurements were performed in ambient air and under $\mathrm{N}_{2}$ atmosphere using an Everbeing EB series probe station with triax cables and probes connected to a Keithley 2450 source measure unit. Additionally, conductance measurements were performed under vacuum using a Desert Cryogenics probe station connected to custom-built electronics. The probe station and triax cables are grounded and function as a Faraday cage. Measurements were conducted in a two-probe or fourprobe configuration. We either applied a fixed voltage bias $\Delta V$ and measured the current $I$ as a function of the time $t$, or alternatively, we performed scans to produce current $(I)$ versus voltage $(V)$ curves (scan rate 0.01 or $0.1 \mathrm{~V} \mathrm{~s}^{-1}$ ). In the former case, the fixed voltage bias was $\Delta V=100 \mathrm{mV}$, and the resistance was determined as $R=\Delta V / I_{\max }$ with $I_{\max }=\max (I[t])$, and the normalized current $I_{\text {norm }}=I_{\max }\left(\Delta x / \Delta x_{\text {ref }}\right)$ with $\Delta x_{\text {ref }}=300 \mu \mathrm{m}$ was calculated to enable a comparison between experiments with different interspacing. When analysing $I / V$ curves, the resistance $R$ was calculated from the slope of the $I / V$ curve at the origin, and the normalized current was calculated as $I_{\text {norm }}=(\Delta V / R)^{*}\left(\Delta x / \Delta x_{\text {ref }}\right)$ with $\Delta V=100 \mathrm{mV}$ and $\Delta x_{\text {ref }}=300 \mu \mathrm{m}$. Electrical noise currents were $<5 \mathrm{pA}$, providing detection limits for single filament conductance $\left(5 \times 10^{-11} \mathrm{~S}\right)$ and fibre conductivity $(0.01 \mathrm{~S}$ $\left.\mathrm{cm}^{-1}\right)$. Test measurements with resistors of known resistance $(100 \mathrm{M} \Omega$ as is the range of filaments) were successfully performed to verify the conductance measurement procedure.

Electrochemical measurements. Cyclic voltammetry at different scan rates and differential pulse voltammetry (step potential $5 \mathrm{mV}$; pulse potential $20 \mathrm{mV}$, pulse time $0.05 \mathrm{~s}$; scan rate $0.01 \mathrm{~V} \mathrm{~s}^{-1}$ ) were conducted in PBS ( $\mathrm{pH} 7.4$ ) buffer using a 3 electrode set-up in a $\mu$ Autolab III electrochemical workstation with a gold BASi electrode (1.6 $\mathrm{mm}$ in diameter) as the working electrode, a glassy carbon rod as the counter electrode and SCE as the reference electrode. Working electrodes were polished successively with 1 and $0.25 \mu \mathrm{m}$ diamond and $0.05 \mu \mathrm{m}$ alumina slurries and were electrochemically treated in $0.5 \mathrm{M} \mathrm{H}_{2} \mathrm{SO}_{4}$ by cyclic potential sweeps from 0.2 to $1.45 \mathrm{~V}$ versus SCE with a scan rate of $0.1 \mathrm{~V} \mathrm{~s}^{-1}$ until a steady-state voltammogram was obtained. Next, the electrodes were incubated for $24 \mathrm{~h}$ in $8 \mathrm{mM}$ mercaptohexanol in MilliQ water, in order to obtain an increased chemical passivation of the electrodes and a minimization of background signals in voltammetry. Prior to use, the electrodes were washed with MilliQ water.

Imaging. Passive Voltage Contrast (PVC) imaging was conducted with a Focused Ion Beam-Scanning Electron Microscope (FIB-SEM). The electron beam was operated at $30 \mathrm{kV}$ with a current of $43 \mathrm{pA}$ and an aperture of $45 \mu \mathrm{m}$, while the ion beam was operated at a current of $10 \mathrm{pA}$. The positive ion beam causes the surface to be positively charged except for areas where an electron sink can remove that charge (i.e. a grounded area), and as a result, the build-up of charge on the surface is inversely correlated to brightness. Filaments were deposited on interdigitated $\mathrm{Au}$ electrodes with $0.5 \mu \mathrm{m}$ non-conductive spacing. Electrodes were grounded by applying carbon paint from the main pad to the back of the chip, which is then in electrical contact with the steel Dual-Beam chamber, effectively grounding the main. Other microscopy imaging methods (equipment and settings) are detailed in the Supplementary Information.

Raman spectroscopy. Resonance Raman spectra of cytochromes were recorded with a Renishaw inVia Reflex Raman microscope with a $\times 50$ objective. A $532 \mathrm{~nm}$ laser was used with $5 \mathrm{~s}$ exposure at $50 \%$ laser power for all measurements. At least 10 individual spectra were recorded from the middle of the filaments to obtain an averaged spectrum. Spectra were subsequently corrected for background scattering from the glass slide and medium by recording additional spectra next to the filaments. Reduced spectra were measured in $10 \mathrm{mM}$ sodium dithionite in artificial seawater (ASW) and oxidized spectra were measured in airsaturated ASW.

Microscopy equipment and settings. Figure 1a The cable bacteria filament was deposited onto the substrate and air-dried. Before imaging, the sample was goldcoated for $30 \mathrm{~s}$, providing a $5 \mathrm{~nm}$-thick gold layer (Polaron E5100 sputter coater, Van Loenen Instruments, Belgium). Scanning electron microscopy (SEM) was performed using a Phenom Pro desktop microscope (Phenom-World B.V., The Netherlands) with a beam intensity of $10 \mathrm{kV}$. Figure $6 \mathrm{a}$. The cable bacteria filamen was deposited onto filter (GF/A $+0.45 \mu \mathrm{m}$ membrane), then folded into holder. The holder was then transferred through a gradual ethanol gradient $(25,50,70$, $90 \%, 2 \times 100 \%$, for $10 \mathrm{~min}$ each), before being critical point dried with liquid carbon dioxide (BALTEC CPD 300, Leica Microsystems, Wetzlar, Germany). Subsequently, specimens were mounted on aluminium stubs (diameter $12 \mathrm{~mm}$ ), and coated with $\sim 50 \mathrm{~nm}$ gold (JEOL JFC-1200 Fine Coater, JEOL, Tokyo, Japan). Images were made with a JEOL JSM-5600 LV (JEOL, Tokyo, Japan) under highvacuum operated at $30 \mathrm{kV}$. Figure $6 \mathrm{~d}$. Specimen were transferred onto Formvar coated copper grids. Samples were transferred in MilliQ water and allowed to airdry on the grids. Imaging was performed on a Tecnai Spirit Electron Microscope at $120 \mathrm{kV}$ using a $4 \times 4 \mathrm{k}$ Eagle camera (Thermo Fischer Scientific, Waltham MA, USA). Figure 7. The cable bacterium sample was placed under a Dino-Lite AM5216ZTL $(10 \times-150 \times)$ camera using a magnification of $\times 30$. An image was recorded using the Dino-Capture 2.0 software, coupling to the Dino-Lite via USB A calibration was done by the software for this single image using the standardized Dino-Lite length scales. Figure $8 \mathrm{a}$. The image was recorded on a brightfield microscope (Olympus BX 51) with a $\times 10$ ocular and $\times 10$ (main image) or $\times 100$ (inset image) objectives, and equipped with a DP25 camera.

Reporting summary. Further information on research design is available in the Nature Research Reporting Summary linked to this article.

\section{Data availability}

Data underlying Fig. 6c, f are provided in the Supplementary Tables 2 and 3, respectively All the other data that support the findings of this study are available from the corresponding author upon reasonable request. 
Received: 26 February 2019 Accepted: 19 August 2019

Published online: 11 September 2019

\section{References}

1. Falkowski, P. G., Fenchel, T. \& Delong, E. F. The microbial engines that drive Earth's biogeochemical cycles. Science 320, 1034-1039 (2008).

2. Marcus, R. A. \& Sutin, N. Electron transfers in chemistry and biology. Biochim. Biophys. Acta 811, 265-322 (1985).

3. Moser, C. C., Keske, J. M., Warncke, K., Farid, R. S. \& Dutton, P. L. Nature of biological electron-transfer. Nature 355, 796-802 (1992).

4. Page, C. C., Moser, C. C., Chen, X. \& Dutton, L. P. Natural engineering principles of electron tunneling in biological oxidation-reduction. Nature 402, 47-52 (1999)

5. Gray, H. B. \& Winkler, J. R. Electron flow through metalloproteins. Biochim. Biophys. Acta 1797, 1563-1572 (2010).

6. Reguera, G. et al. Extracellular electron transfer via microbial nanowires. Nature 435, 1098-1101 (2005)

7. Gorby, Y. A. et al. Electrically conductive bacterial nanowires produced by Shewanella oneidensis strain MR-1 and other microorganisms. Proc. Natl Acad. Sci. USA 103, 11358-11363 (2006).

8. El-Naggar, M. Y. et al. Electrical transport along bacterial nanowires from Shewanella oneidensis MR-1. Proc. Natl Acad. Sci. USA 107, 18127-18131 (2010).

9. Nielsen, L. P., Risgaard-Petersen, N., Fossing, H., Christensen, P. B. \& Sayama, M. Electric currents couple spatially separated biogeochemical processes in marine sediment. Nature 463, 1071-1074 (2010).

10. Pfeffer, C. et al. Filamentous bacteria transport electrons over centimeter distances. Nature 491, 218-221 (2012).

11. Bjerg, J. T. et al. Long-distance electron transport in individual, living cable bacteria. Proc. Natl Acad. Sci. USA 115, 5786-5791 (2018).

12. Nielsen, L. P. \& Risgaard-Petersen, N. Rethinking sediment biogeochemistry after the discovery of electric currents. Annu. Rev. Mar. Sci. 7, 425-442 (2015).

13. Meysman, F. J. R. Cable bacteria take a new breath using long-distance electricity. Trends Microbiol. 26, 411-422 (2018).

14. Malkin, S. Y. et al. Natural occurrence of microbial sulphur oxidation by longrange electron transport in the seafloor. ISME J. 8, 1843-1854 (2014)

15. Burdorf, L. D. W. et al. Long-distance electron transport occurs globally in marine sediments. Biogeosciences 14, 683-701 (2017).

16. Risgaard-Petersen, N. et al. Cable bacteria in freshwater sediments. Appl. Environ. Microbiol. 81, 6003-6011 (2015).

17. Müller, $\mathrm{H}$. et al. Long-distance electron transfer by cable bacteria in aquifer sediments. Isme. J. 10, 2010-2019 (2016).

18. Schauer, R. et al. Succession of cable bacteria and electric currents in marine sediment. ISME J. 8, 1314-1322 (2014).

19. Risgaard-Petersen, N., Damgard, L. R., Revil, A. \& Nielsen, L. P. Mapping electron sources and sinks in a marine biogeobattery. J. Geophys. Res.: Biogeosci. 119, 1475-1486 (2014).

20. Meysman, F. J. R., Risgaard-Petersen, N., Malkin, S. Y. \& Nielsen, L. P. The geochemical fingerprint of microbial long-distance electron transport in the seafloor. Geochim. Cosmochim. Acta 152, 122-142 (2014).

21. Cornelissen, R. et al. The Cell Envelope Structure of Cable Bacteria. Front. Microbiol. 9, 3044 (2018)

22. Jiang, Z. et al. In vitro single-cell dissection revealing the interior structure of cable bacteria. Proc. Natl Acad. Sci. USA 115, 8517-8522 (2018).

23. Reimers, C. E., Li, C., Graw, M. E., Schrader, P. S. \& Wolf. M. The identification of cable bacteria attached to the anode of a benthic microbial fuel cell: evidence of long distance extracellular electron transport to electrodes. Front. Microbiol. 8, 2055 (2017).

24. Vasquez-Cardenas, D. et al. Microbial carbon metabolism associated with electrogenic sulphur oxidation in coastal sediments. ISME J. 9, 1-13 (2015).

25. Owen, J. in Comprehensive Polymer Science and Supplements (eds. Allen G. \& Bevington J. C.) vol 2, 669-686 (1996).
26. Polizzi, N. F., Skourtis, S. S. \& Beratan, D. N. Physical constraints on charge transport through bacterial nanowires. Farad. Disc. 155, 43-62 (2012).

27. Adhikari, R. Y., Malvankar, N. S., Tuominen, M. T. \& Loveley, D. R. Conductivity of individual Geobacter pili. RSC Adv. 6, 8354-8357 (2016).

28. Lampa-Pastirk, S. et al. Thermally activated charge transport in microbial protein nanowires. Sci. Rep. 6, 23517 (2016).

29. Le, T.-H., Kim, Y. \& Yoon, H. Electrical and electrochemical properties of conducting polymers. Polymers 9, 150 (2017).

\section{Acknowledgements}

This research was financially supported by the European Research Council under the European Union's Seventh Framework Programme (FP/2007-2013) through ERC Gran 306933 (F.J.R.M.), the Research Foundation Flanders (FWO project grant G031416N), and the Netherlands Organisation for Scientific Research (VICI grant 016.VICI.170.072 to F.J.R.M.). H.J.E.B., C.J.B. and H.S.J.Z. were supported by the Netherlands Organisation for Scientific Research (NWO/OCW), as part of the Frontiers of Nanoscience program R.B. is supported by an 'aspirant' grant from Research Foundation Flanders (FWO). We thank Laurine Burdorf (UAntwerpen) for help with Thiothrix cultivation, Marlies Nijemeisland (Faculty of Aerospace, TU Delft) for assistance with Raman microscopy, and Jan D'Haen (UHasselt) and Renaat Dasseville (UGent) for help with EM imaging.

\section{Author contributions}

F.J.R.M. designed and coordinated the study. S.H.M. and J.S.G. cultivated cable bacteria and Thiofilum flexile. S.H.M. performed filament retrieval and extraction. C.J.B., H.J.E.B., and H.S.J.Z. conducted wafer probe analysis and PVC imaging on filament bundles. R.C., R.B., J.H., R.T.E., B.C., R.V, H.T.S.B. and J.V.M. conducted probe station analysis on single filaments of cable bacteria under ambient air and $\mathrm{N}_{2}$. J.v.d.V. and H.S.J.Z. conducted probe station analysis on fibre sheaths under vacuum. C.K., S.T. and K.d.W. performed cyclic voltammetry. H.T.S.B. performed resonance Raman spectroscopy. F.J.R.M. wrote the manuscript and all other authors gave input and feedback.

\section{Additional information}

Supplementary Information accompanies this paper at https://doi.org/10.1038/s41467019-12115-7.

Competing interests: The authors declare no competing interests.

Reprints and permission information is available online at http://npg.nature.com/ reprintsandpermissions/

Peer review information Nature Communications thanks Leonard Tender and the other, anonymous, reviewer(s) for their contribution to the peer review of this work. Peer reviewer reports are available.

Publisher's note Springer Nature remains neutral with regard to jurisdictional claims in published maps and institutional affiliations.

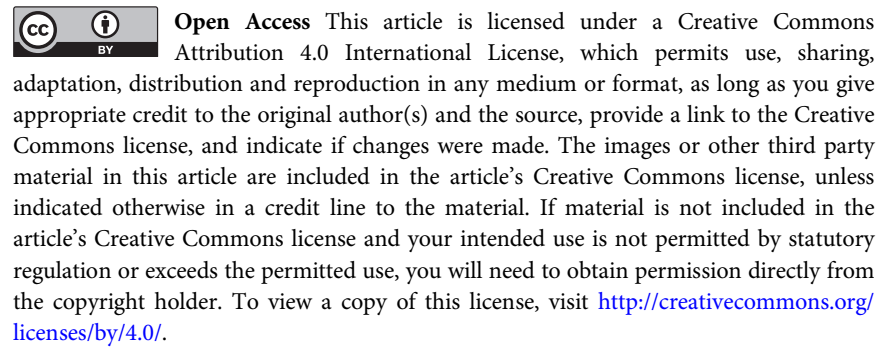

(C) The Author(s) 2019 